\title{
Contaminación del aire y salud respiratoria en niños
}

Mireia Gascon ${ }^{1,2,3,4}$, Jordi Sunyer ${ }^{2,3,4,5}$

${ }^{1}$ ISGlobal, Barcelona Ctr. Int. Health Res. (CRESIB), Hospital Clínic - Universitat de Barcelona, Barcelona, Spain

${ }^{2}$ Centre for Research in Environmental Epidemiology (CREAL), Barcelona, Catalonia, Spain.

${ }^{3}$ Universitat Pompeu Fabra (UPF), Barcelona, Catalonia, Spain.

${ }^{4}$ CIBER Epidemiología y Salud Pública (CIBERESP), Spain.

${ }^{5}$ IMIM (Hospital del Mar Medical Research Institute), Barcelona, Catalonia, Spain.

\section{Autor de correspondencia}

Mireia Gascon

Barcelona Biomedical Research Park (PRBB) - Centre for Research in Environmental Epidemiology (CREAL)

Doctor Aiguader, 88 | 08003 Barcelona, Spain

Phone: $0034932147353 \quad$ Fax: 0034932045904

email: mgascon@creal.cat

Número de palabras: 782

\section{Agradecimientos y financiación}

No se ha recibido ningún tipo de financiación para la realización de este manuscrito 
En marzo de 2014 la Organización Mundial de la Salud (OMS) alertaba que la contaminación del aire, que incluye contaminantes como el material particulado (siglas PM en inglés), el ozono $\left(\mathrm{O}_{3}\right)$, el dióxido de nitrógeno $\left(\mathrm{NO}_{2}\right)$ o el dióxido de azufre $\left(\mathrm{SO}_{2}\right)$, causa anualmente 3.7 millones muertes prematuras en todo el mundo ${ }^{1}$. De hecho, la contaminación del aire, en concreto el material particulado, es la primera causa ambiental de muerte y enfermedad, y la novena causa absoluta por delante del colesterol o la falta de ejercicio físico ${ }^{2}$. En los últimos años varios estudios sobre los efectos agudos y crónicos de los contaminantes del aire han confirmado que el riesgo de padecer accidentes cerebrovasculares, cardiopatías, cáncer de pulmón, y enfermedades respiratorias crónicas y agudas, incluyendo el asma, incrementa a mayor exposición a estos contaminantes ${ }^{1}$. Es más, parece ser que no existe un umbral de seguridad y que incluso a exposiciones muy bajas ya podría haber efectos en la salud ${ }^{3}$.

La cohorte de parejas madre-niño del proyecto INMA (INfancia y MedioAmbiente) ${ }^{4}$ ha contribuido al conocimiento científico en este sentido centrándose en los efectos de la exposición a contaminantes del aire durante la vida prenatal y su papel en la incidencia de enfermedades respiratorias y en el desarrollo del pulmón. En el 2013 Aguilera et al., en un estudio que incluía más de 2.000 participantes provenientes de cuatro ciudades españolas, observaron que el riesgo de padecer infecciones de las vías respiratorias inferiores y otitis a la edad de un año era mayor en aquellos niños cuyas madres estaban expuestas a mayores concentraciones de contaminantes provenientes del tráfico $\left(\mathrm{NO}_{2} \mathrm{y}\right.$ benceno) durante el embarazo ${ }^{5}$. Posteriormente estos mismos datos se combinaron con datos de otras seis cohortes de nacimiento Europeas. De nuevo, se observó que la exposición prenatal a $\mathrm{NO}_{2}$, y también otros contaminantes del aire como el $\mathrm{PM}_{10}$, incrementaban el riesgo de padecer pneumonia y/o otitis durante 
los primeros dos años de vida ${ }^{6}$. Otro trabajo que incluía 74.000 recién nacidos de once cohortes encontró una disminución del peso al nacer en relación a la contaminación por tráfico a nivel de domicilio, con las consiguientes implicaciones sobre el desarrollo pulmonar $^{7}$. Recientemente, un estudio que incluye datos de las dos cohortes INMA que midieron función pulmonar temprana, con un alto porcentaje de curvas de buena calidad, describió que la $\mathrm{FEV}_{1}$ de los niños a la edad de 4.5 años disminuía a mayores niveles de exposición a $\mathrm{NO}_{2}$ y benceno durante el embarazo ${ }^{8}$. A pesar de que los efectos eran pequeños a nivel individual los resultados confirman el papel de la contaminación del aire y sus efectos tempranos, que podrían estar relacionados con la aparición de la enfermedad pulmonar obstructiva crónica (EPOC) a largo plazo ${ }^{9}$.

Estos resultados, consistentes entre sí y con la literatura disponible hasta el momento, son importantes si tenemos en cuenta que a nivel mundial las infecciones respiratorias agudas representan el 20\% de las muertes anuales en niños menores de 5 años, siendo el porcentaje mayor en países en desarrollo donde los niveles de contaminantes del aire son mucho mayores que en Europa ${ }^{1}$. Aunque se considera que el aire limpio es un requisito básico de la salud y el bienestar humanos, los estudios demuestran que su contaminación sigue representando una amenaza importante para la salud en todo el mundo y que la mejora de la calidad del aire debería estar en las agendas políticas de todos los países. Lamentablemente, la nueva Comisión Europea está considerando dar marcha atrás con la directiva impulsada en el 2013 que pretendía reducir la contaminación proveniente de instalaciones de combustión medianas y una revisión de los límites de emisión a la baja en los países miembros ${ }^{10}$, unos límites ( $25 \mu \mathrm{g} / \mathrm{m}^{3}$ para $\mathrm{PM}_{2.5}$ ) que actualmente ya están por encima de los establecidos por la OMS (10 $\left.\mu \mathrm{g} / \mathrm{m}^{3}\right)^{10}$. Vistos los resultados que demuestran el alto impacto de la contaminación del 
aire sobre la salud respiratoria - un 14\% de las muertes causadas por la contaminación del aire son debidas a problemas respiratorios - la comunidad de neumólogos, como ya hizo con el tabaco, debería actuar como lobby para presionar a las autoridades competentes en Europa y que reconsideren la decisión de aumentar los niveles permitidos. Igualmente, los neumólogos pueden jugar un papel de presión ante las respectivas autoridades locales que deben implementar las medidas adecuadas para que se cumplan los criterios de calidad del aire que ayudan a proteger la salud. Como profesionales de la salud y como sociedad debemos asegurar una mayor calidad de vida a todos nuestros conciudadanos, especialmente la de los niños y niñas, más sensibles a la exposición a estos contaminantes ambientales.

\section{Agradecimientos y financiación}

No se ha recibido ningún tipo de financiación para la realización de este manuscrito.

\section{Bibliografía}

1. World Health Organization. Ambient (outdoor) air quality and health. Fact sheet N³13. World Health Organization 2014; [consultado 03-02-2015]: Disponible en: http://www.who.int/mediacentre/news/releases/2014/air-pollution/en/

2. Lim SS, Vos T, Flaxman AD, Danaei G, Shibuya K, Adair-Rohani H, et al. A comparative risk assessment of burden of disease and injury attributable to 67 risk factors and risk factor clusters in 21 regions, 1990-2010: a systematic analysis for the Global Burden of Disease Study 2010. Lancet 2012;380:2224-60.

3. Lancet. Air pollution: Europe’s avoidable health risk. Lancet 2013;381:876.

4. Guxens M, Ballester F, Espada M, Fernandez MF, Grimalt JO, Ibarluzea J, et al. Cohort Profile: The INMA--INfancia y Medio Ambiente--(Environment and Childhood) Project. Int J Epidemiol. 2012;41:930-40.

5. Aguilera I, Pedersen M, Garcia-Esteban R, Ballester F, Basterrechea M, Esplugues A, et al. Early-life exposure to outdoor air pollution and respiratory health, ear infections, and eczema in infants from the INMA study. Environ Health Perspect. 2013;121:38792. 
6. MacIntyre EA, Gehring U, Mölter A, Fuertes E, Klümper C, Krämer U, et al. Air pollution and respiratory infections during early childhood: an analysis of 10 European birth cohorts within the ESCAPE Project. Environ Health Perspect. 2014;122:107-13.

7. Pedersen M, Giorgis-Allemand L, Bernard C, Aguilera I, Andersen A-MN, Ballester F, et al. Ambient air pollution and low birthweight: a European cohort study (ESCAPE). Lancet Respir Med. 2013;1:695-704.

8. Morales E, Garcia-Esteban R, de la Cruz OA, Basterrechea M, Lertxundi A, de Dicastillo MDML, et al. Intrauterine and early postnatal exposure to outdoor air pollution and lung function at preschool age. Thorax 2015;70:64-73.

9. Sly PD. Traffic-related air pollution: an avoidable exposure to improve respiratory health. Thorax 2015;70:3-4.

10. The Lancet Respiratory Medicine. Pollution control up in the air. Lancet Respir Med 2015;3:87. 